\title{
Podemos atuar preventivamente para evitar que os pacientes portadores de COVID-19 evoluam de forma mais grave?
}

\author{
Can we manage prophylactic therapy in COVID-19 patients to prevent \\ severe illness complications? \\ Paulo Eduardo Ocke Reis ${ }^{1}$ (D), Marcos Cesar Braga Lima
}

\begin{abstract}
Resumo
Muitos pacientes com COVID-19 apresentam complicaç̃es tromboembólicas que acabam piorando seu prognóstico. Os autores propõem uma modificação no escore $\mathrm{CHA}_{2} \mathrm{DS}_{2}$-VASc, incluindo 1 ponto para COVID-19, para, desse modo, indicar profilaxia de eventos tromboembólicos antes do agravamento do quadro. As vantagens dessa modificação seriam evitar a piora do paciente por problemas tromboembólicos, bem como a necessidade de internação em unidade de tratamento intensivo e de ventilação mecânica, e diminuir a mortalidade.
\end{abstract}

Palavras-chave: COVID-19; coronavirus; anticoagulantes; trombose; prevenção; mortalidade hospitalar.

\begin{abstract}
Many patients with COVID-19 have thromboembolic complications that worsen their prognosis. Herein, the authors propose a modified version of the $\mathrm{CHA}_{2} \mathrm{DS}_{2}-\mathrm{VASC}$ score, including 1 point for COVID-19, so that prophylaxis to protect against thromboembolic events would be indicated before the condition becomes severe. The advantages of this modification would be prevention of the patient's condition worsening due to thromboembolic problems and reduction of the likelihood of a need for intensive care and mechanical ventilation, reducing mortality.
\end{abstract}

Keywords: COVID-19; coronavirus; anticoagulants; thrombosis; prevention; hospital mortality.

Como citar: Reis PEO, Lima MCB. Podemos atuar preventivamente para evitar que os pacientes portadores de COVID-19 evoluam de forma mais grave? J Vasc Bras. 2020;19:e20200057. https://doi.org/10.1590/1677-5449.200057 


\section{INTRODUÇÃO}

Desde a primeira descrição da infecção por COVID-19, a síndrome respiratória grave associada à doença levou ao aumento rápido de admissões em unidades de tratamento intensivo (UTIs) e à alta mortalidade de um grupo de pacientes ${ }^{1}$. Em uma pandemia, é preciso evitar a saturação do sistema de saúde tanto público quanto privado, em especial das UTIs. O principal achado com relevância nos pulmões é a presença de trombos plaquetários e fibrina em pequenos vasos arteriais, enquadrando-se perfeitamente no contexto clínico da coagulopatia ${ }^{2}$.

Como não há um tratamento aprovado em consenso neste cenário e tendo em vista a possibilidade de trombose associada à infecção pelo coronavírus em determinados casos, a experiência adquirida recentemente e estudos científicos ainda embrionários têm mostrado que uma anticoagulação efetiva poderia prevenir ou reverter o estado pró-trombótico de alguns pacientes ${ }^{2,3}$.

\section{PROPOSTA}

Observamos que, coincidentemente, o grupo de pacientes que evoluem mal da infecção por COVID-19 (Figura 1) ${ }^{4}$ e morrem são os mesmos pacientes do escore $\mathrm{CHA}_{2} \mathrm{DS}_{2}$-VASc com risco para acidente vascular cerebral, episódio isquêmico transitório, embolia periférica e tromboembolismo pulmonar (Tabela 1$)^{5,6}$. Por esse escore, o paciente é considerado de alto risco se a pontuação for $2 \mathrm{ou}$ mais, de risco intermediário se a pontuação for $1 \mathrm{e}$ de baixo risco se não tiver fatores de risco ${ }^{6}$. A nossa proposta, portanto, é incluir 1 ponto adicional no escore $\mathrm{CHA}_{2} \mathrm{DS}_{2}$-VASc (Tabela 1) para pacientes portadores de COVID-19 e usar o novo escore para indicar a anticoagulação profilática nos pacientes com alto risco de trombose pelo escore, na fase $2 \mathrm{da}$ doença (Tabela 2). Desse modo, busca-se evitar a piora do paciente por problemas tromboembólicos, bem como a necessidade de internação em UTI e de ventilação mecânica ${ }^{7}$.

A ideia é agir de forma similar ao risco de tromboses e embolias pelos escores conhecidos e iniciar a profilaxia para tentar evitar a ocorrência do que tem contribuído para o agravamento do quadro clínico desses pacientes ${ }^{1-3}$. Através deste artigo, os autores propõem uma modificação pontual na escore $\mathrm{CHA}_{2} \mathrm{DS}_{2}$-VASc para estudo de sua validação, com o objetivo de diminuir o número de doentes críticos que chegam à fase 3 .

Taxa de mortalidade

Idade

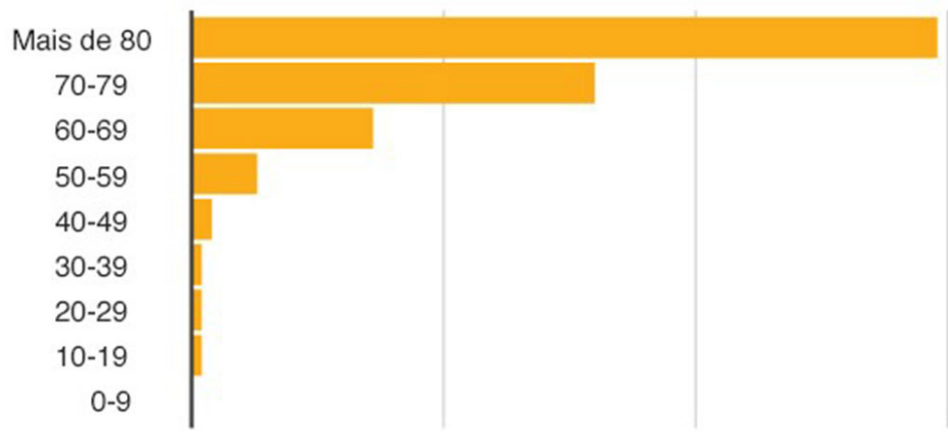

\section{Condição de saúde}

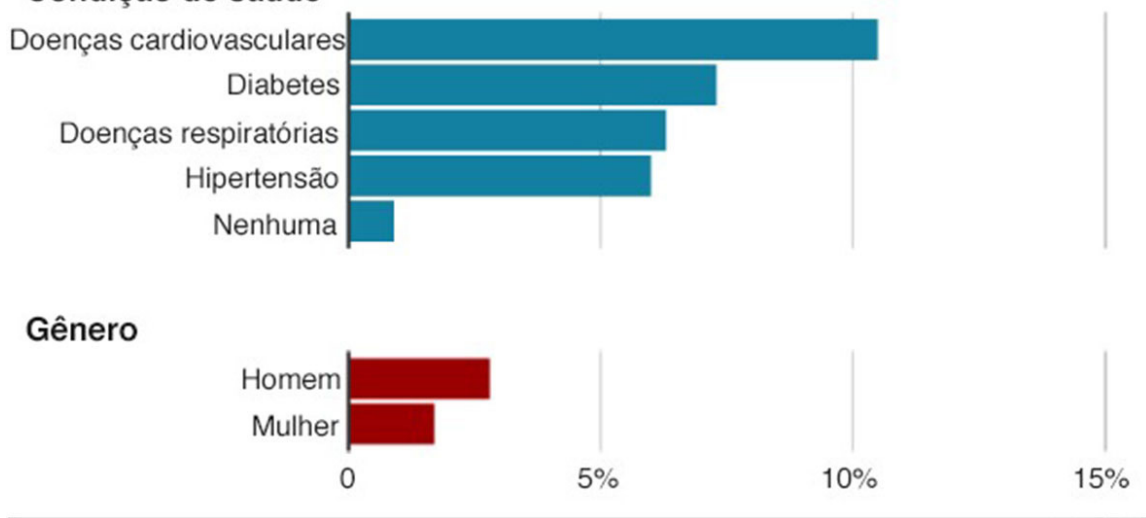

Figura 1. A taxa de mortalidade por COVID-19 varia de acordo com a idade e a condição de saúde 4 . 
Tabela 1. Como ficaria o escore $\mathrm{CHA}_{2} \mathrm{DS}_{2}$-VASc com o acréscimo de 1 ponto por COVID-19 (CHA $\mathrm{DS}_{2}$-VASc-C19).

\begin{tabular}{ccc}
\hline CHA $_{2}$ DS $_{2}$-VASc & Descrição & Pontos \\
\hline C & Insuficiência cardíaca & 1 \\
$\mathrm{H}$ & Hipertensão & 1 \\
$\mathrm{~A}_{2}$ & Idade $(\geq 75$ anos) & 2 \\
$\mathrm{D}$ & Diabetes mellitus & 1 \\
$\mathrm{~S}_{2}$ & AIT ou AVC prévio & 2 \\
$\mathrm{~V}$ & Doença vascular (IAM prévio, placa aórtica, doença arterial periférica) & 1 \\
$\mathrm{~A}$ & Idade (65-74 anos) & 1 \\
$\mathrm{C} 19$ & Suspeita ou confirmação de COVID-19 & 1 \\
\hline
\end{tabular}

AIT = ataque isquêmico transitório; $A V C$ = acidente vascular cerebral; IAM = infarto agudo do miocárdio.

Tabela 2. Fases da infecção por COVID-19 e tratamento.

\begin{tabular}{cll}
\hline Fases & \multicolumn{1}{c}{ Clínica } & \multicolumn{1}{c}{ Tratamento } \\
\hline Fase 1 & $\begin{array}{l}\text { Infecção respiratória } \\
\text { gripal }\end{array}$ & $\begin{array}{l}\text { Evitar contágio, diminuir sintomas, diminuir carga viral com as medicações que } \\
\text { estão sendo usadas }\end{array}$ \\
Fase 2 (verificar Tabela 1) & Alto risco de trombose & $\begin{array}{c}\text { Profilaxia, evitar trombose intrapulmonar, anticoagulação profilática } \\
\text { Fase 3 }\end{array}$ \\
\hline
\end{tabular}

UTI = unidade de tratamento intensivo.

\section{REFERÊNCIAS}

1. Huang C, Wang Y, Li X, et al. Clinical features of patients infected with 2019 novel coronavirus in Whuam, China. Lancet. 2020;395(10223):497-506. http://dx.doi.org/10.1016/S01406736(20)30183-5. PMid:31986264.

2. Casarna L, Sonzogni A, Nasr A, et al. Pulmonary post-mortem findings in a large series of COVID-19 cases from Northern Italy. medRxiv; 2020 [citado 2020 abr 28]. https://www.medrxiv.org/ content/10.1101/2020.04.19.20054262v1

3. Obi AT, Barnes GD, Wakefield TW, et al. Practical diagnosis and treatment of suspected venous thromboembolism during COVID-19 Pandemic. J Vasc Surg Venous Lymphat Disord. 2020. http://dx.doi.org/10.1016/j.jvsv.2020.04.009. PMid:32305585.

4. Cuffe R. Coronavírus: quais as chances de morrer por causa da covid-19. Brasil: BBC News; 2020 [citado 2020 abr 28]. https:// www.bbc.com/portuguese/internacional-51703189

5. Sá T, Sargento-Freitas J, Pinheiro V, et al. CHADS e CHA $\mathrm{DS}_{2}$ VASC como preditores de fonte cardioembólica em prevenção secundária cerebrovascular. Rev Port Cardiol. 2013;32(5):373-8. http://dx.doi. org/10.1016/j.repc.2012.09.007. PMid:23566635.

6. Habboushe J, Altman C, Lip GYH. Time trends in use of the $\mathrm{CHADS}_{2}$ and $\mathrm{CHA}_{2} \mathrm{DS}_{2}$ VASc scores, and the geographical and specialty uptake of these scores from a popular online clinical decision tool and medical reference. Int J Clin Pract. 2019;73(2):e13280. http:// dx.doi.org/10.1111/ijcp.13280. PMid:30281876.

7. Bikdeli B, Madhavan MV, Jimenez D, et al. COVID-19 and thrombotic or thromboembolic disease: implications for prevention, antithrombotic therapy, and follow-up. J Am Coll Cardiol. 2020;17:272-84. PMid:32311448.
Correspondência Paulo Eduardo Ocke Reis Universidade Federal Fluminense - UFF, Departamento de Cirurgia Geral e Especializada Rua Marques de Paraná, 303 - Centro CEP 24033-900 - Niterói (RJ), Brasil Tel.: (21) 2629-5000 E-mail:vascular@pauloocke.com.br; ockereis@yahoo.com

Informações sobre os autores PEOR - MD; PhD; Especialista em Cirurgia Vascular e Endovascular; Professor Adjunto de Cirurgia Vascular, Universidade Federal Fluminense (UFF); Doutorado, Universidade Federal do Rio de Janeiro (UFRJ).

MCBL - MD; Especialista em Cardiologia, Universidade Federal Fluminense (UFF); Diretor, Clínica Cardiológica Dr. Marcos Cesar Braga Lima.

Contribuições dos autores Concepção e desenho do estudo: PEOR, MCBL Análise e interpretação dos dados: PEOR, MCBL Coleta de dados: PEOR Redação do artigo: PEOR, MCBL Revisão crítica do texto: PEOR, MCBL Aprovação final do artigo*: PEOR, MCBL Análise estatística: MCBL Responsabilidade geral pelo estudo: PEOR, MCBL

*Todos os autores leram e aprovaram a versão final submetida ao J Vasc Bras. 